\title{
Cutaneous Post Inflammatory Hyperpigmentation from Morsicatio Buccarum
}

\author{
Alexis E Carrington, ${ }^{1 *}$ Marjon Vatanchi ${ }^{2}$ \\ ${ }^{1}$ Department of Dermatology, University of California Davis School of Medicine, USA \\ ${ }^{2}$ Department of Dermatology, The Warren Alpert Medical School at Brown University, USA
}

\begin{abstract}
Post Inflammatory hyperpigmentation (PIH) is a pigmentary phenomenon which commonly follows disease or injury. Examples include thermal or caustic burns, infections, or inflammatory disorders affecting the skin such as acne, atopic dermatitis, or rash. PIH can occur in anyone but is more common in Fitzpatrick types III-VI individuals, where the color tends to be more intense and persist for a longer period than in Fitzpatrick types I and II individuals. We present a unique case of PIH on the skin as a result of frequent biting of the inner buccal mucosa.

Keywords: Pigmentation, PIH, Oral, Biting, Morsicatio buccarum
\end{abstract}

\section{Case Report}

A 19-year-old healthy Caucasian man presented to the dermatology office for hyperpigmentation on the cheeks for several years. He never wore sunscreen and denied any external factors, trauma, or history of rash. He denied any pain or pruritus of the mouth or cheeks. He admitted to a habit of biting the inside of his mouth due to mild anxiety. On examination, there was faint brown hyperpigmentation on bilateral medial cheeks lateral to the oral commissures. On oral examination, there were white plaques with stippling at bite sites inside the mouth. When the plaques were pressed upon inside the mouth, it aligned directly with the sites of hyperpigmentation. Patient was diagnosed with PIH from morsicatio buccarum biting the buccal mucosa. Treatment included strict avoidance of buccal biting, stress management, daily sunscreen application, and sun avoidance.

\section{Discussion}

PIH is an acquired disorder characterized by excess deposition of melanin in the epidermis, dermis, or both occurring secondary to cutaneous inflammation or injury. ${ }^{2}$ Although the exact mechanism of PIH remains unclear, it is postulated that inflammatory cells release cytokines and other mediators which are capable of stimulating melanocytes and increasing melanin synthesis. ${ }^{3}$

Melanocytes are found throughout the oral mucosa but usually go unnoticed because of their relatively low level of pigment production. ${ }^{4}$ Oral pigmentation can be caused from physiologic pigmentation, systemic diseases, drug reactions or malignant neoplasms. PIH in the oral mucosa is commonly secondary to fixed drug reactions, commonly presenting as well-demarcated areas of hyperpigmentation commonly affecting the oral mucosa and lips. ${ }^{5}$ So far, thirty cases of hyperpigmentation of the oral mucosa, skin and nails have occurred secondary to imatinib. ${ }^{6}$

A careful examination of the oral cavity may reveal the first manifestation of underlying systemic diseases. When the diagnosis cannot be reached clinically with enough certainty, a biopsy for histological examination is required, especially if malignancies such as melanoma are in the differential. ${ }^{4}$ Most cases of PIH are self-resolving although this can be lengthy and distressing for the patient.

\begin{tabular}{l|l}
\hline Quick Response Code: & *Corresponding author: Alexis E Carrington, Department of Dermatology, University of Cali- \\
fornia Davis School of Medicine, 3301 C Street, Suite 1300, Sacramento, CA 95816, USA
\end{tabular}


For treatment, formulations of tretinoin, adapalene and tazarotene have all shown efficacy in treating epidermal causes of PIH, with one study finding tretinoin $0.004 \%$ microsphere gel the least irritating in pigmented skin. ${ }^{2}$ Chemical peels and lasers can be utilized by an expert dermatologist with experience treating pigmentary

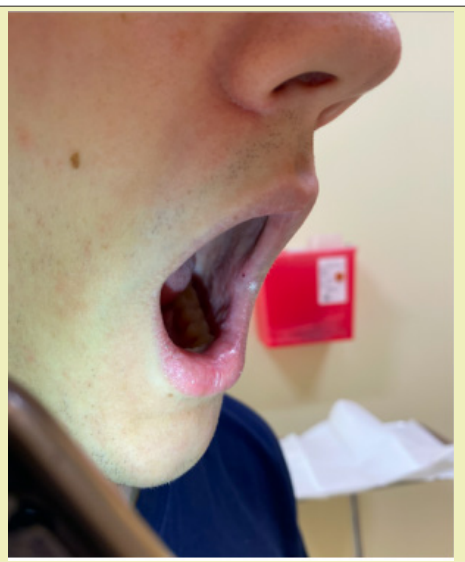

Figure 1: Left buccal mucosa showing evidence of morsicatio buccarum.

\section{Acknowledgments}

None.

\section{Funding}

None.

\section{Conflicts of Interest}

No conflicts of interests to disclose.

\section{References}

1. Lamel, S. Post inflammatory hyperpigmentation secondary to external insult: an overview of the quantitative analysis of pigmentation. Cutan Ocul Toxicol. 2013;32(1):67-71. disorders; all these procedures carry a risk of hyperpigmentation. Cutaneous PIH due to morsicatio buccarum has not been described in the literature until now. The trauma induced by chronic biting of the buccal mucosa can activate melanocytes on the skin surface to produce faint hyperpigmentation.

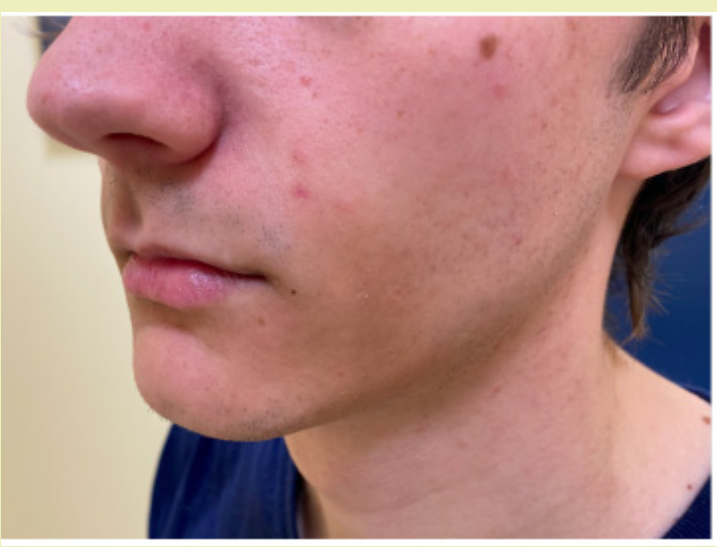

Figure 2: Arcuate hyperpigmented patch on the left medial cheek.

2. Geria, A. Topical retinoids for pigmented skin. J Drugs Dermatol. 2011;10(5):483-489.

3. Morelli J, Norris D. Influence of inflammatory mediators and cytokines in human melanocyte function. JInvest Dermatol.1993;100:191S-195S.

4. Lambertini M. Oral pigmentation in physiologic conditions, post-inflammatory affections and systemic diseases. Giornale Italiano di Dermatologia e Venereologia. 2018;153(5):666-671.

5. Craig LH. Pigmented lesions of the oral cavity. Dent Clin North Am. 2005;49(1):185-201.

6. Di Tullio. Imatinib-induced diffuse hyperpigmentation of the oral mucosa, the skin, and the nails in a patient affected by chronic myeloid leukemia: report of a case and review of the literature. Intl Jour Derm. 2018.57(7):784-790. 